\title{
Measurement of Elastic Velocities of MgO under Shock Compression to 500 Kilobars
}

\author{
Geoffrey F. Davies ${ }^{1}$ and Thomas J. Ahrens
}

\author{
Seismological Laboratory, California Institute of Technology, Pasadena, California 91109
}

The velocities of rarefaction waves in shock-compressed $\mathrm{MgO}$ have been measured by observing the reduction of the shock front velocity near the sample edges due to the rarefaction waves propagating from the edges. The extent of this 'edge effect' is difficult to determine accurately because of its emergent nature. Arrangements sensitive to differences in shock front velocity yielded rarefaction wave velocities close to predicted longitudinal velocities in the high-pressure shock state. Velocities closer to the hydrodynamic sound speed in the shock state were obtained from less sensitive arrangements. These results can be interpreted in terms of a two-stage elastoplastic model of the decompression. The longitudinal velocities measured in shock states up to $528 \mathrm{~kb}$ imply second pressure derivatives of the elastic moduli $c_{i j^{\prime \prime}}$, given by $K_{0} c_{i j}{ }^{\prime \prime}=-1 \pm 15$, where $K$ is the bulk modulus.

The measurement of the velocities of rarefaction waves that propagate into shockcompressed materials provides a method for directly determining the elastic properties of the material in the high-pressure shock state. Direct measurement of the elastic moduli of solids, using ultrasonic techniques, have to date been made only to pressures of about $20 \mathrm{~kb}$. At higher pressures, information about elasticity is usually obtained from pressure-density curves determined by static compression $\mathrm{X}$ ray, piston cylinder measurements, or from shock wave Hugoniot data. These determinations yield only one elastic modulus, the bulk modulus, and considerable accuracy is lost because the derivative of the pressure-density data has to be taken. Alternative, more direct methods of determining the elastic moduli are therefore potentially very useful. Initial results are reported here of measurements of rarefaction wave velocities in $\mathrm{MgO}$ shock-compressed to over $500 \mathrm{~kb}$.

\section{EXPERIMENTAL TECHNIQUE}

The method used in this study consists of measuring the extent of the rarefaction that propagates into the shock region from the sides of the sample. Such a method was applied by

\footnotetext{
${ }^{1}$ Now at the Hoffman Laboratories, Harvard University, Cambridge, Massachusetts 02138.
}

Copyright (C) 1973 by the American Geophysical Union.
Al'tshuler et al. [1960] to the study of some metals. The configuration of the sample and the waves is shown in Figure 1. The shock wave is generated at the lower surface of the sample as a result of the impact of a projectile whose velocity is measured just prior to impact [Ahrens et al., 1971]. As the shock wave propagates through the sample, rarefactions propagate inward from the unconstrained (zero pressure) sides of the sample, reducing the shock pressure and hence reducing the velocity of the shock front near the edge of the sample. The combined lower shock velocity and lower pressure give rise to a lower free surface velocity near the sample edges when the shock front reaches the top sample surface. A mirror is placed a small distance above the sample with the silvered surface facing the sample, and the impact of the sample onto the mirror is recorded by viewing the mirror through a slit, the image of which is streak-recorded by an image converter camera (Figure 2) [Ahrens et $a l ., 1971]$. Typical records are shown in Figure 3 . The cutoff of the film streak, schematically illustrated in Figure 2, occurs first in the central part, corresponding to that part of the shock front unaffected by lateral rarefactions. The cutoff then occurs progressively further toward the edges of the sample as the slower moving free surface nearer the edges reaches the mirror. The extent of the edge effect is determined by measuring the central linear portion of the cutoff. 


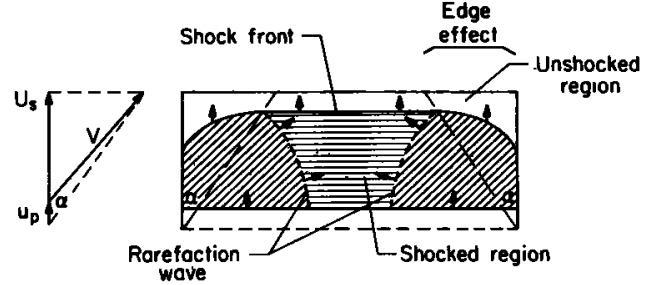

Fig. 1. Configuration of the shock and rarefaction waves produced by passage of a shock wave from the lower surface of the sample. Arrows show the geometrical relationship between the rarefaction wave velocity $V$, the particle velocity $u_{p}$, and the shock front velocity $U_{\text {. }}$.

The first rarefaction signal to reach an interior point is that propagating from the lower corners of the crystal. Simple geometrical relations (Figure 1) then give the rarefaction velocity $V$ as

$$
V=U_{a}\left[\tan ^{2} \alpha+\left(U_{c}-u_{p}\right)^{2} / U_{a}^{2}\right]^{1 / 2}
$$

where $U_{\mathrm{s}}$ is the shock front velocity, $u_{\mathrm{p}}$ is the particle velocity, and $\alpha$ is the angle between the side of the sample and the locus of intersection of the shock and rarefaction waves (Figure 1).

The sample is mounted on a tungsten 'driver plate' that is struck by a tungsten 'flyer plate' mounted in the tip of the projectile. The pressure $P$ and particle velocity in the sample are determined by impedance matching [e.g., Rice et al., 1958], using the measured projectile velocities and $P-u_{p}$ curves for tungsten [McQueen

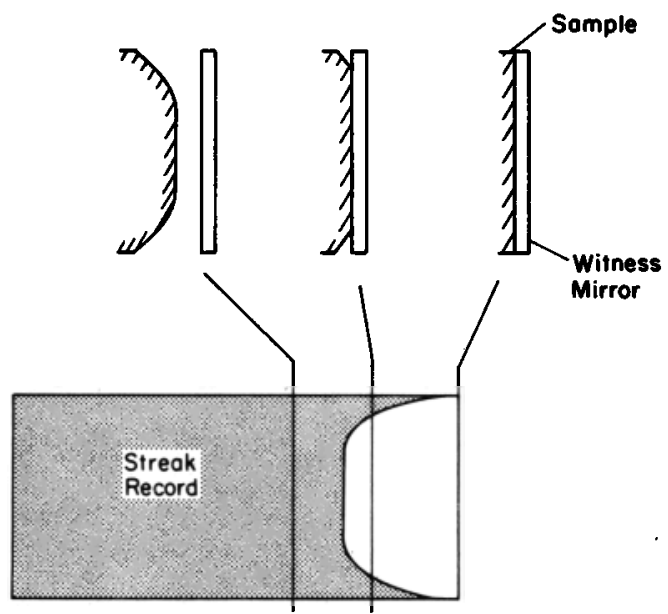

Fig. 2. Schematic illustration of the recording by the streak camera of the impact of the sample on the witness mirror.

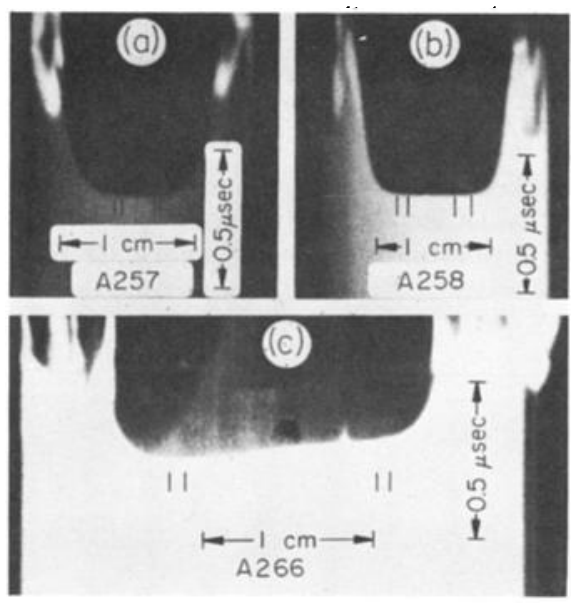

Fig. 3. Streak records of sample-mirror impacts. Length (horizontal) and time (vertical) scales are indicated. Numbers refer to shot numbers (Table 1). Initial mirror-sample separations were (a) $0.76 \mathrm{~mm}$, (b) $0.13 \mathrm{~mm}$, (c) $0.25 \mathrm{~mm}$. Samples $a$ and $b$ were polycrystalline, and sample $c$ was single crystal.

et al., 1970] and $\mathrm{MgO}$ [Carter et al., 1971]. The shock velocity is then calculated from the Rankine-Hugoniot relation

$$
U_{.}=P / \rho_{0} u_{p}
$$

where $\rho_{0}$ is the zero-pressure density of the sample.

The $\mathrm{MgO}$ samples used in this study were five polycrystalline samples supplied by $T$. Vasilos of the Avco Corporation and three single crystals with (100) cleavage faces purchased from the Norton Research Corporation. The polycrystalline samples described and measured ultrasonically by Schreiber and Anderson [1968] and Spetzler [1970] were obtained from the same source.

\section{ResulTs}

A basic difficulty of this method is the emergent nature of the edge effect (Figure 3). Initial experiments were therefore performed with the mirror spaced a small distance $(0.76 \mathrm{~mm})$ from the sample surface. This spacing allows the contrast in free surface velocities across the sample to amplify the contrast in the total transit time, which is the sum of the shock wave transit time through the sample and the free surface transit time to the mirror. Sample specifications and results for four such shots are 
given in Table 1. Figure $3 a$ (shot A257) is a typical record. Since there was not a really welldefined flat central section of the cutoff in these records (variations of light extinction over 10 nsec existed over the central $2 \mathrm{~mm}$ of the sample), it was thought that some deformation of the sample free surface might be occurring during the transit. To test this idea, a shot (A258, Figure $3 b$ and Table 1) was fired with the mirror-sample separation reduced to 0.13 $\mathrm{mm}$. (The spacing was not reduced to zero to avoid any complications from the elastic precursor to the shock wave [e.g., Ahrens, 1966].) $A$ well-defined flat central section of the cutoff was obtained (Figure $3 b$ ), but the measured rarefaction velocity was considerably lower than that in the previous shots (Table 1). The remaining three shots were fired on the singlecrystal samples with intermediate mirror-sample spacing $(0.25 \mathrm{~mm})$. Results are given in Table 1 and Figure $3 c$ and exhibit rarefaction velocities intermediate between those of the previous shots. (The origin of the low-angle irregularities in shot A266, shown in Figure $3 c$, which were also observed in the other single-crystal shots, is unknown. Some uncertainty in the extent of the edge effect results from their presence.)

\section{Discussion}

An interpretation of these observations is suggested by the observations of other workers. Al'tshuler et al. [1960] observed that the edge effect had a much sharper beginning for shocked liquids than for shocked solids and that the measured rarefaction velocities corresponded closely to the hydrodynamic (bulk) sound speeds for the liquids but that for the solids they were higher and corresponded more closely to the expected longitudinal elastic velocities of the solids. Kusubov and van Thiel [1969] observed the decompression of 6061-T4 aluminum directly using manganin pressure gages and found that the decompression occurred in two stages, the first stage propagating at about the velocity of longitudinal elastic waves and the second stage, identified by an increase in the rate of decompression, propagating at about the bulk sound speed of aluminum. These observations were interpreted in terms of a two-stage elastoplastic decompression, in which the decompression is one dimensional and elastic until a critical deviatoric stress is reached, after

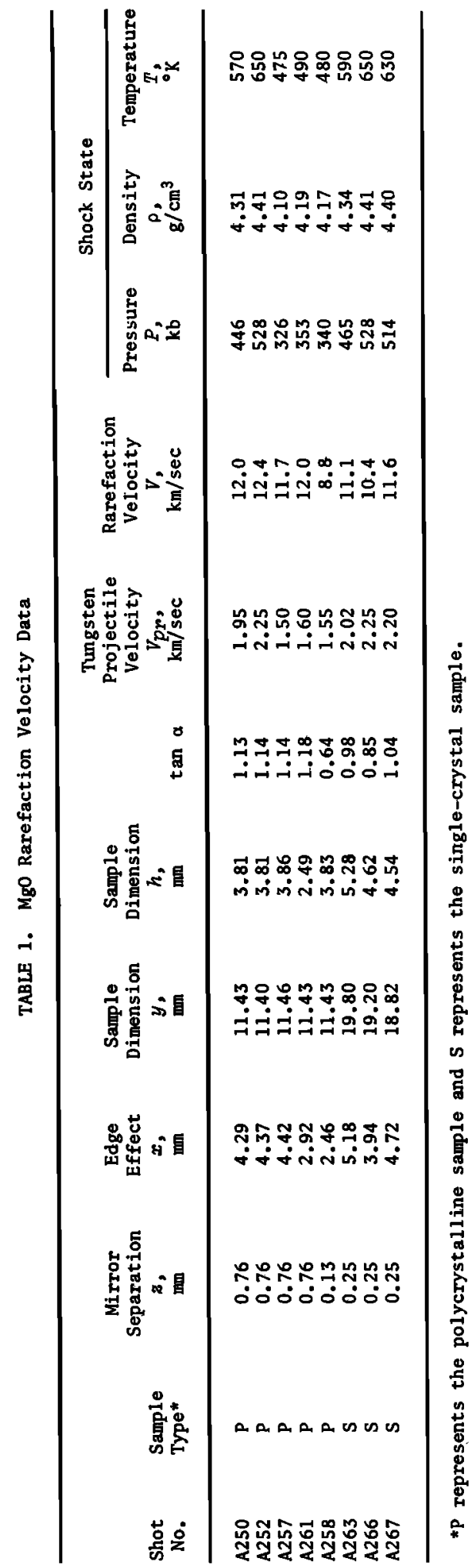


which it is approximately plastic. The reverse process has been observed in the shock compression of $\mathrm{MgO}$ [Ahrens, 1966] and other materials.

The interpretation of the present observations is aided by comparisons between the measured velocities and various extrapolations of the longitudinal elastic velocity $V_{L}$ and the bulk sound speed $C$ (which are discussed in detail below). The measured and extrapolated velocities are plotted against pressure in Figure 4. The shots with the greatest mirror-sample separation $z$, which were the most sensitive to contrasts in free surface velocities, yielded the highest measured rarefaction velocities. These velocities are close to the extrapolations of $V_{\mathbf{L}}$. The shots with smaller $z$ yielded considerably lower measured rarefaction velocities closer to $C$ for the smallest value of $z$ (A258). This finding suggests that the arrangement in the latter shots was not sensitive enough to detect the initial elastic decompression observed in the previous shots, so that the observed edge effect was produced mainly by the hydrodynamic (or plastic) decompression. (The half error bars shown in Figure 4 were obtained by picking points on the records where edge effects definitely existed. The best estimates of the extent of the edge effect and the lower bounds are indicated by the inner and outer parts of tick marks in Figure 3, respectively. Obviously, upper bounds cannot be estimated.)

The major uncertainty in the above interpretation of the data is whether the profile of the free surface when it strikes the witness mirror accurately reflects the conditions at the free surface when the shock wave reached it or whether the free surface undergoes some distortion during transit to the witness mirror. Suspicions of the latter effect were in fact the original reason for varying the mirror-sample spacing. Finite difference computations are being carried out in an attempt to verify the source of the observed effects.

The extrapolations of $V_{L}$ and $C$ in Figure 4 are based on the elastic moduli and their pressure and temperature derivatives determined ultrasonically by Spetzler [1970]. They are third- and fourth-order finite strain extrapolations in terms of the Eulerian and Lagrangian strain parameters $\epsilon$ and $\eta$ and include the thermal effects in the 'quasi-harmonic' approxi-

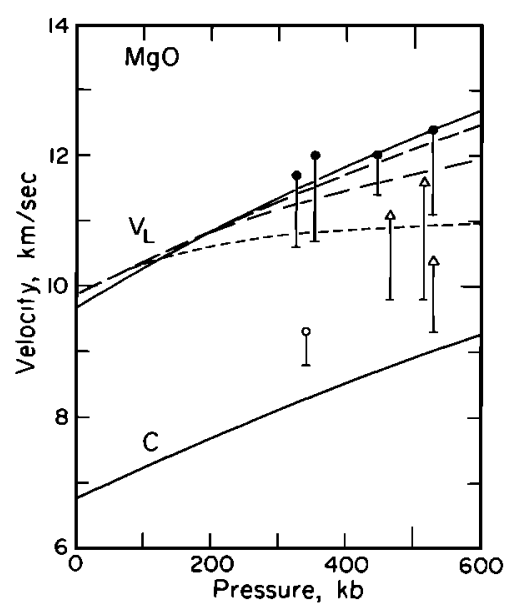

Fig. 4. Measured rarefaction velocities and extrapolated longitudinal $\left(V_{L}\right)$ and bulk $(C)$ sound velocities in the shocked state. Mirrorsample separations were $\mathbf{0 . 7 6} \mathrm{mm}$ (solid circles), $0.13 \mathrm{~mm}$ (open circle), and $0.25 \mathrm{~mm}$ (triangles). Extrapolations are for polycrystal, $\zeta_{i j}=-1$ (solid); single-crystal [110] direction, $\zeta_{1 j}=-1$ (long-dashed); $\zeta_{110}=-10$ (dash-dot); and thirdorder Lagrangian (short-dashed).

mation [Davies, 1972; unpublished manuscript, 1973]. In terms of $\epsilon$, the fourth-order finite strain formula for the effective elastic moduli $c_{i}$ (Voigt notation) from which the velocities are calculated has the form [Davies, 1972; unpublished manuscript, 1973]

$$
\begin{aligned}
c_{i j}= & \rho_{0}(1-2 \epsilon)^{7 / 2} \\
& \cdot\left(r_{i i}{ }^{0}+r_{i j}{ }^{1} \epsilon+\frac{1}{2} r_{i}{ }^{2} \epsilon^{2}\right)-P \Delta_{i j}
\end{aligned}
$$

where the $r_{i j}{ }^{n}$ are parameters, $P$ is the pressure, and, in the case of cubic symmetry,

$$
\Delta_{11}=-3 \quad \Delta_{12}=-1 \quad \Delta_{44}=-1
$$

The corresponding expression for the pressure is [Davies, 1972, 1973]

$$
P=-\frac{1}{3} \rho_{0}(1-2 \epsilon)^{5 / 2}\left(c_{0}+c_{1} \epsilon+c_{2} \epsilon^{2}+c_{3} \epsilon^{3}\right)
$$

The strain parameter $\epsilon$ is related to density by

$$
\epsilon=\frac{1}{2}\left[1-\left(\rho / \rho_{0}\right)^{2 / 9}\right]
$$

The parameters $c_{n}$ and $r_{i}{ }^{n}$ in (3) and (5) are related to the $c_{\imath}$ and their pressure derivatives evaluated at $\rho_{0}$. These relations are given in the appendix. Analogous equations in terms of the Lagrangian strain $\eta$ can also be derived [Davies, 
1972, 1973, unpublished manuscript, 1973; Thomsen, 1970, 1972].

The full fourth-order versions of (3) and (5) require the second pressure derivatives of the effective elastic moduli $c_{i j}$ " (see equation A7). These have not been measured, but the present results can be used to place constraints on them. The second pressure derivative of the bulk modulus $K^{\prime \prime}$ of $\mathrm{MgO}$ has been determined from shock wave data to be such that $\zeta=K_{0} K_{0}^{\prime \prime}=$ -1 [Davies, 1973]. As a first approximation it was assumed that $\xi_{i j}=K_{0} c_{i j}{ }^{\prime \prime}=-1$. The resulting extrapolations of the polycrystalline average velocities $V_{L}$ and $C$ are shown in Figure 4 as solid curves. Note that these extrapolations include corrections for the increasing temperature along the Hugoniot (Table 1). This extrapolation of $V_{L}$ gives good agreement with the measurements obtained by using the largest mirror-sample separation (solid circles). The appropriate longitudinal velocity in the singlecrystal samples is that for the [110] crystallographic direction, since $\tan \alpha \simeq 1$ (Table 1) and the crystal faces were (100) planes. This velocity $V_{110}$ is shown extrapolated in Figure 4 by the long-dashed curve. It is quite close to the polycrystalline velocity, and the distinction is hardly warranted by the accuracy of the data.

The bounds on the $c_{i j}$ " can be estimated from the extrapolation of $V_{110}$ by using $\zeta_{110}=1 / 2$ $\left(\zeta_{11}+\zeta_{12}+2 \zeta_{44}\right)=-10$, which is shown as the dash-dot curve. We may thus deduce that $\xi_{v}=-1 \pm 15$ where $\zeta_{v}$ is the appropriate combination of $\zeta_{1}$, (i.e., corresponding to the polycrystalline or the [110] single-crystal velocity).

In view of the current discussion of the relative empirical merits of the $\eta$ and $\epsilon$ strain measures [e.g., Ahrens and Thomsen, 1972], third-order $\eta$ and $\epsilon$ extrapolations of $V_{L}$ were compared with the present data. The thirdorder $\epsilon$ extrapolation was found to be quite close to the fourth-order $\epsilon$ extrapolation with $\zeta_{i j}=-1$ and is not shown in Figure 4. The third-order $\eta$ extrapolation is shown by the short-dashed curve. It is seen to give a poorer fit to the solid circles, which are here interpreted as the most accurate measurements of $V_{L}$.

In conclusion, our data suggest that the two-stage elastoplastic model for solids [e.g., Kusubov and van Thiel, 1969] applies to $\mathrm{MgO}$ under decompression. This implies also that
$\mathrm{MgO}$ remains in the solid state to at least 500 $\mathrm{kb}$ and $600^{\circ} \mathrm{K}$ under shock compression. Our results yield the approximate bounds $\zeta_{0}=$ $-1 \pm 15$ as determined from Eulerian finite strain equations. Further refinement of this technique should allow more accurate determinations of the high-pressure, high-temperature elastic properties of materials of geophysical interest.

\section{APPENDIX}

The parameters $c_{n}$ in (5) are related to the $r_{i j}{ }^{n}$ in $(3)$ :

$$
\begin{aligned}
& c_{1}=3\left(r_{11}{ }^{0}+2 r_{12}{ }^{0}\right) \\
& c_{2}=\frac{3}{2}\left(r_{11}{ }^{1}+2 r_{12}{ }^{1}\right) \\
& c_{3}=\frac{1}{2}\left(r_{11}{ }^{2}+2 r_{12}{ }^{2}\right)
\end{aligned}
$$

By evaluating (5) at $\rho_{0}$, we see that

$$
c_{0}=-3 P_{0} / \rho_{0}
$$

where $P_{0}$ is the pressure at $\rho_{0}$, and $P_{0}$ depends on the temperature $T$.

By differentiating (3), the parameters $r_{i j}{ }^{n}$ can be related to $P_{0}$ and the $c_{i j}$ and their pressure derivatives $c_{i j}{ }^{\prime}, c_{i j^{\prime \prime}}$, etc., evaluated at $\rho_{0}$ :

$$
\begin{aligned}
r_{i j}{ }^{0} & =\left(c_{i j}+P_{0} \Delta_{i j}\right) / \rho_{0} \\
r_{i i}{ }^{1} & =-3 K_{0}\left(c_{1}{ }^{\prime}+\Delta_{i j}\right) / \rho_{0}+7 r_{i i}{ }^{0} \\
r_{1 i}{ }^{2} & =9 K_{0}{ }^{2} c_{i j}{ }^{\prime \prime} / \rho_{0}-3 K_{0}{ }^{\prime}\left(r_{i}{ }^{1}-7 r_{i j}{ }^{0}\right) \\
& +16 r_{i j}{ }^{1}-49 r_{i i}{ }^{0}
\end{aligned}
$$

where $K=1 / 3\left(c_{11}+2 c_{12}\right)$ is the bulk modulus.

Thermal effects in the finite strain equations 3 and 5 can be described through the parameters $r_{i j}{ }^{n}$ and $c_{n}$. Expressions for the temperature dependence of these parameters have been derived [Davies, 1972, 1973, unpublished manuscript, 1973] from the quasi-harmonic approximation of lattice dynamics [Leibfried and Ludwig, 1961]. These expressions require for their evaluation the thermal expansion coefficient, the temperature derivatives of the elastic moduli, and the specific heat of the material.

Acknowledgments. We appreciate the help of D. Johnson and $\mathrm{H}$. Richardson in performing the experiments. We thank $T$. Vasilos of the Avco Corporation for providing the polycrystalline samples. 
This research was supported by National Science Foundation grant GA-21396. Contribution 2275, Division of Geological and Planetary Sciences, California Institute of Technology, Pasadena, California 91109.

\section{REFERENCES}

Ahrens, T. J., High-pressure electrical behaviour and the equation of state of magnesium oxide from shock wave measurements, J. Appl. Phys., 37, 2532-2541, 1966.

Ahrens, T. J., and L. Thomsen, Application of the fourth-order anharmonic theory to the prediction of equations of state at high compressions and temperatures, Phys. Earth Planet. Interiors, 5, 282-294, 1972.

Ahrens, T. J., J. H. Lower, and P. L. Lagus, Equation of state of forsterite, J. Geophys. Res., 76, 518-528, 1971.

Al'tshuler, L. V., S. B. Kormer, M. I. Brazhnik, L. A. Vladimirov, M. P. Sperenskaya, and A. I. Funtikov, The isentropic compressibility of aluminum, copper, lead and iron at high pressures, J. Exp. Theor. Phys., 88, 1061-1073, 1960.

Carter, W. J., S. P. Marsh, J. N. Fritz, and R. G. McQueen, The equation of state of selected materials for high-pressure references, Accurate Characterization of the High Pressure Environment, edited by E. C. Lloyd, Nat. Bur. Stand. Spec. Publ. $32 b, 1971$.

Davies, G. F., Elasticity of solids at high temperatures and pressures, Ph.D. thesis, pp. 6697, Calif. Inst. of Technol., Pasadena, 1972.
Davies, G. F., Quasi-harmonic finite strain equations of state of solids, J. Phys. Chem. Solids, 34, 1417-1429, 1973.

Kusubov, A. S., and M. van Thiel, Measurement of elastic and plastic unloading wave profiles in 2024-T4 aluminum alloy, J. Appl. Phys., 40, 3776-3780, 1969.

Leibfried, G., and W. Ludwig, Theory of anharmonic effects in crystals, Solid State Phys., 12, 275-444, 1961.

McQueen, R. G., S. P. Marsh, J. W. Taylor, J. N. Fritz, and W. J. Carter, The equation of state of solids from shock wave studies, in High Velocity Impact Phenomena, edited by R. Kinslow, pp. 294-419, Academic, New York, 1970.

Rice, M. H., R. G. McQueen, and J. M. Walsh, Compression of solids by strong shock waves, Solid State Phys., 6, 1-63, 1958.

Schreiber, E., and O. L. Anderson, Revised data on polycrystalline magnesium oxide, $J$. Geophys. Res., 79, 2837-2838, 1968.

Spetzler, H., Equation of state of polycrystalline and single-crystal $\mathrm{MgO}$ to 8 kilobars and $800^{\circ} \mathrm{K}$, J. Geophys. Res., 75, 2073-2087, 1970.

Thomsen, L., On the fourth-order anharmonic equation of state of solids, J. Phys. Chem. Solids, 31, 2003-2016, 1970.

Thomsen, L., The fourth-order anharmonic theory: Elasticity and stability, J. Phys. Chem. Solids, 3s, 363-378, 1972.

(Received January 10, 1973; revised July 20, 1973.) 